\title{
INTERNET AS AN INSTRUMENT TO TRANSMIT THEORETICAL KNOWLEDGE
}

\author{
Svetlana Kvesko ${ }^{1}$, Svetlana Kvesko ${ }^{2}$, Nataliya Kabanova ${ }^{3}$, Daria Shamrova $^{4}$ \\ ${ }^{1}$ National Research Tomsk State University, 634050, Tomsk, Russia \\ ${ }^{2}$ Lyceum № 10, Krasnoyarsk, 660113, Russia \\ ${ }^{3}$ National Research Tomsk Polytechnic University, 634050, Tomsk, Russia \\ ${ }^{4}$ Michigan State University, 49254, Michigan, USA
}

\begin{abstract}
The problem of transmitting theoretical knowledge and the role of the Internet in it require the solution due to the existing modernization of theoretical knowledge transmission process. The objective of this research is to define the role of the Internet in transmitting theoretical knowledge as it is the extremely important resource of the modern society. According to the carried out analysis of the problem and its solution the information technology is not only the mean that accumulates the volumes of knowledge, but also the tool of its social use, forms of social activity by way of social and information technology. As a result, using method of the methodological analysis in combination with competency-based approach we revealed that the Internet as a diachronic way of transmitting knowledge and experience is characterized by a polyagentity and interdisciplinarity.
\end{abstract}

\section{Introduction}

The history of society is the history of transmitting theoretical and practical knowledge and experience from a person to a person, from a generation to a generation, from one epoch to another. This is the history of transmitting society's social memory (sociocode). Knowledge and experience transmission can be of various methods [1]:

1. Transmission is carried out either by means of address communication, during communicative practice, or through the address communication having operational character. The activity of people as individuals in social environment of the cohabitation and joint activity is coordinated during such communication. This kind of communication is called synchronous. Transmission of both empirical and theoretical knowledge is carried out by means of synchronous communication.

2. Information and experience can be transferred trough time, i.e. from generation to generation, from epoch to epoch, here is transmission of knowledge and experience occurs. Such way is called diachronic.

These two ways differ from each other in character of feedback communication. At synchronous transmitting knowledge, information is known to both subjects of educational process, while at diachronic communication, the information is known to one subject. The

"Corresponding author: svetla_kvesko@mail.ru 
knowledge is generally connected with transmission, with diachronic communication namely. Generally, theoretical knowledge is connected with transmission.

Both synchronous, and diachronic ways of transmitting knowledge use language, sign reality, as the main information storage and transmission devices. We should note that here also artificial languages are used, which that is connected with use of information and measuring equipment and technology.

\section{Method}

The aim of this research is to define the role of the Internet in transmitting theoretical knowledge as an extremely significant resource of modern society, as not a mean for accumulating the scope of knowledge only, but the instrument of its social use, as a form of social activity in social and information technology. The method of the methodological analysis in accordance with a competence-based approach is applied to detect necessary characteristics of transmitting theoretical knowledge.

\section{Main content}

Transmitting knowledge is the unity of objective and subjective knowledge expressed through language: either natural, or artificial, and through writing. The objective knowledge is kept in various material forms, including computer memory, books etc. We deal with an object in information stored in objective knowledge, for example, in computer memory. The object has not been interpreted and transformed in speech. In this case objective knowledge is a part of the social activity presented in signs. This objective knowledge is theoretical knowledge which is objective according to its content.

However, degree of adequacy of percepting information by means of transmission (diachronic communication) depends on a subject and personal form of theoretical knowledge and its cover. The subjective knowledge represents language model of the situation which is subjective and individual and personal expression of language of the broadcast theoretical knowledge [2]. This knowledge is a result of systematization of statements, theses, provisions, the points of view.

Adequacy and extent of closeness to the objective content of theoretical knowledge depends on experience, a variety of relationships of a personality with culture of society, intelligent potential of an individual. And as a result, methods of interpretation and formalization get importance. Interpretation broadens the sense-conceptual field of information, but formalization promotes developing forms of transmitting theoretical knowledge.

Transmission of theoretical knowledge is carried out when a neutrality is, and there is no any personal influence, exact reflection. The language picture of the world is the picture of the world reflecting the world by means of languages both natural, and artificial. The last one is characteristic of the Internet.

The Internet as the instrument of transmitting theoretical knowledge is based on use of technology of communication practice when the knowledge is addressed to a great number of addressees.

Transmitting theoretical knowledge is connected with a defined type of society. Preinformation epoch societies paid much attention to the teacher/lecturer, imparting knowledge to pupils/trainee. In information society information technology including the Internet, have impact on various forms of activity in society and, certainly, on transmitting theoretical knowledge. As a result, theoretical knowledge transforms to information resource. Its storage, processing and transmission to the subject of informative process is 
provided with information technology which allow to obtain, process and transmit great deal of information at high speed [3].

Computer storage devices give an opportunity to form a synchronous way of transmitting knowledge in addition to a diachronic way. Consequently, the information system of theoretical knowledge is being formulated, which is able to coordinate the process transmitting the sum of knowledge in connection with address communication [4].

The unity of communication (synchronous way) and transmission (diachronic way) causes feedback, i.e. provides correction of knowledge transmission programs. As the essence of transmission is transmission of theoretical knowledge from one part having information to the other part not having it, therefore, the Internet becomes the instrument of transmission.

Theoretical knowledge transmission via the Internet is based on communication technologies, on the principles of synchronism, results in increase of erudition and education of a person, extent of modern society intellectualization.

Theoretical knowledge transmission under the mass use of the Internet has no clear boundaries, and is not controlled practically, and consequently it leads to polyagent (multilateral) and interdisciplinary use. The absence of information selection criteria and its variety complicate the use of interdisciplinary approach and a polyagentity in assimilating theoretical knowledge received via the Internet.

We can claim that the universal and conceptual way of a person's inclusion to social reality and bank of theoretical knowledge via the Internet is an essential elements of using computer technologies in information space by a person for work.

In the 21 st century information transmission becomes not a simply important and essential resource. This leads to updating computer technologies and the Internet as the instrument of transmitting information. As a result, expansion of using electronic resource brings about the emergence of various developed, multilateral (polyagent) social networks, which are characterized by existence of interdisciplinary communications. All these factors cause forms of people social activity, such as social and humanitarian technologies together with computer.

Theoretical knowledge, being abstract and deep on its essence, explains the phenomena, processes, and events. It is widely used by a person to familiarize in social and natural environment, to forecast and draw up a road map.

Due to the accelerated information and computer technologies development, their intensification, the innovative forms of electronic learning occurs, which assume training people via the Internet. Such method of studying allows to higher intellectualization of modern society, increasing intellectual potential of citizens [5]. However, great deal of information, various assessments of theoretical objectives, the giving uncontrolled standpoints complicate formulating a clear picture of event, making the selection of significant theoretical knowledge difficult.

The method of transmitting theoretical knowledge via Internet transmission provides disregarding qualities, skills and opportunities of a particular personality. Such knowledge transmission influence formulating world outlook, lines o thoughts, value system. Such theoretical knowledge transmission is informal as it tends to a certain convention, blurring, and multilateral interpretation of the gained knowledge [6]. On the Internet it is possible to receive any knowledge by means of a discourse. Searching and developing new ways of transmission had lead to informal methods of processing, understanding, assimilating greater content of theoretical knowledge.

The solution of the problem of theoretical knowledge transmission, the question of speed and volumes of the imparted theoretical knowledge via the Internet is important for revealing specifics and features of computer and information technology development 
influence on processes in society and in the nature, on language and culture, on education and upbringing.

Increase in speed of transmitting knowledge is caused by formalization of communication language that leads to creation of universal language [7].

\section{Conclusion}

Having studied the essence of the Internet as instrument of transmitting theoretical knowledge we received the following results:

1. We revealed that transmitting knowledge is a unity of both objective and subjective knowledge.

2. Theoretical knowledge transmission is carried out when there is neutrality, and there is no any personal influence, precise reflection. $\mathrm{f}$

3. The Internet as the instrument of transmitting theoretical knowledge is based on the use of communication practice technology when knowledge is addressed to a great number of addresses.

4. It has been proved that computer data storages give an opportunity to form a synchronous way of transmitting knowledge in addition to a diachronic way. As a result, the information system of theoretical knowledge is formulated, which is able to coordinate the process of transmitting the sum of knowledge in connection with address communication.

5. It has been founded that the unity of communication (a synchronous way) and transmission (a diachronic way) causes feedback, i.e. provides correction of knowledge transmission programs.

6. The essence of transmission is definitely the transmitting of theoretical knowledge from a having information part, to the part which has not it; the Internet here is the instrument of transmission.

7. We have revealed that transmitting theoretical knowledge in the context of the Internet mass use has no distinct limits, and it is basically not controllable. Consequently it leads to polyagent (multilateral) and interdisciplinary use.

8. We have established that due to the lack of information criteria selection, and owing to its variety the use of interdisciplinary approach and a polyagentity in digestion of theoretical knowledge received from the Internet is complicated.

9. It is arguable that the universal and conceptual way of a person's inclusion to the social reality and theoretical knowledge bank via the Internet is an essential element of using computer technology by a person to work in information space.

10. We emphasize that there is an updating of computer technology and the Internet as an instrument of transmitting information. As a result, the widening usage of an electronic resource brings into existence an extremely developed, various, multilateral (polyagent) social networks which are also characterized by the existence of interdisciplinary communications.

11. We have proved that the emergence of the innovative forms of electronic learning assuming people training via the Internet results from the accelerated development of information and computer technologies and their intensification.

According to the carried-out methodological analysis based on polyagent and interdisciplinary approaches we can draw conclusions:

Information technology is the cornerstone of modern economy. This new economy is based on knowledge. The technology of generating knowledge makes essence of productivity in modern economy. 
Transmission of theoretical knowledge, qualitatively developing. as a way of knowledge transmission, is capable to give an impulse to changes in economy, culture, education and in other spheres of life.

Information technologies represent a new form of social activity. They act as disambiguation of a contradiction between growth of volume of knowledge and social use of information technology.

The diachronic way of transmitting theoretical knowledge, which is closely connected with the Internet as the transmission instrument, led to emergence of electronic learning, i.e. the learning based on using the Internet.

Language is used in transmitting theoretical knowledge as the main social sign reality. It should be noted that there is a problem of creation of universal language.

Improvement of process of understanding of the broadcast knowledge acts as an urgent task in a solution of the problem of transfer and processing of theoretical knowledge by means of use of the Internet as the tool.

The problem of transmitting theoretical knowledge and the role of the Internet in it require the solution due to the existing modernization of the process of transmitting theoretical knowledge. As it appears from the undertaken study the information technology is the instrument of social use of theoretical knowledge transmission. Therefore we may state that the Internet represents a diachronic way of transmitting knowledge.

\section{Acknowledgment}

This research is carried out under the scope "Organization and methodic issues of training specialists in Information and Measurement Technologies and Technique". Passes with assistance of a grant of the Russian fund of basic researches №16-07-20218, and the program of improving competitiveness of the TSU (project No. 8.2.31.2015).

\section{References}

[1] V.L. Anoshkina, S.V. Rezvanov, Education. Innovation. Future. Methodological and sociocultural problems (Publishing house of RO IPK and PRO,Rostov-on-Don, 2001)

[2] M. Porter. Harvard Business Review 76 , 77 (1998).

[3] N. Kachalov, A. Kornienko, R. Kvesko, Yu. Nikitina, S. Kvesko, Zh. Bukharina, Procedia Social and Behavioral Sciences 206, $459 \quad$ (2015) doi: $10.1016 /$ j.sbspro.2015.10.083

[4] I.B. Ardashkin, R.B. Kvesko, O.M. Ardashkina IFOST-2013 2, 692 (2013)

[5] M. Lehmann, P. Christensen, X. Du \& M. Thrane. EJEE. 33 , 283 (2008)

[6] H. Etzkowitz, L. Leydesdorff, Research Policy 29, 243 (2000)

[7] V.I. Syryamkin, E.G. Syryamkina, Procedia Social and Behavioral Sciences 166, $471(2015)$ 